COMUNICAÇÃO CIENTÍFICA

\title{
RESPOSTA DE DIFERENTES GENÓTIPOS DE TANGERINAS E SEUS HÍBRIDOS À INOCULAÇÃO IN VITRO E IN VIVO DE Alternaria alternata ${ }^{1}$
}

\author{
FERNANDO ALVES AZEVEDO², DENIS AUGUSTO POLYDORO³, MARINES BASTIANEL ${ }^{2}$, \\ KATIA CRISTINA KUPPER ${ }^{2}$, RODRIGO MAKOWIECKY STUART ${ }^{2}$, \\ FRANCELINO PEREIRA COSTA ${ }^{4}$, ROSE MARY PIO²
}

RESUMO - A severidade da mancha-marrom de alternária nos pomares brasileiros de tangerinas tem causado sérias preocupações aos citricultores, devido aos prejuízos em plantios comerciais de variedades suscetíveis, como a tangerina Ponkan e o tangor Murcott. Para avaliar a resposta de diferentes genótipos ao fungo, foram realizadas inoculações de Alternaria alternata in vitro e in vivo, em 54 diferentes genótipos de tangerinas e seus híbridos, selecionados no Banco Ativo de Germoplasma de Citros do Centro APTA Citros Sylvio Moreira, do Instituto Agronômico, em Cordeirópolis-SP, visando a encontrar variedades resistentes. Para isso, inicialmente, testes de patogenicidade foram realizados com dez isolados de A. alternata para a seleção dos mais agressivos. Posteriormente, foram realizadas inoculações em folhas destacadas e em plântulas e, aos dois (in vitro) e três (in vivo) dias após, fez-se a contagem do número de lesões/folha e a estimativa da severidade da doença com auxílio de escala diagramática (in vivo). A maior parte dos genótipos apresentou sintomas da doença, porém com diferentes graus de suscetibilidade. Genótipos como a tangerina Sul da África e o tangelo Orlando foram os mais suscetíveis. Por outro lado, o grupo das satsumas e mexericas, assim como algumas tangerinas mostraram-se resistentes, indicando novas opções para a citricultura nacional. Termos para indexação: mancha-marrom de Alternária, Citrus reticulata, resistência varietal.

\section{RESPONSE OF DIFFERENT TANGERINE VARIETIES AND HYBRIDS TO IN VITRO AND IN VIVO INOCULATION OF Alternaria alternata}

\begin{abstract}
The confirmation of a new disease in the Brazilian tangerine orchards, the alternaria brown spot, has been concerning citrus growers because of the serious damages to commercial crops of susceptible varieties, such as Ponkan tangerine and Murcott tangor. In this research, in vitro and in vivo inoculations of Alternaria alternata fungus were held in 54 different varieties of the tangerine group selected from the Citrus Germoplasm Bank of the Citros Center APTA Sylvio Moreira, from the Instituto Agronomico, in Cordeirópolis, State of São Paulo, Brazil, aiming to find varieties with more resistance. First, a pathogenicity test with ten different $A$. alternata isolates was made to select the most aggressive one. After this, inoculations in detached leaves and seedlings were held and, approximately two (in vitro) and three (in vivo) days after, the number of lesions in each leaf was counted, and the disease severity estimated with diagrammatic scale help (in vivo). More than a half of varieties showed symptoms of the disease, however with different levels of susceptibility. Varieties like South African tangerine and Orlando tangelo were the most susceptible; on the other hand the satsumas and willow leaf group and some tangerines, showed resistance, indicating new options to the Brazilian citriculture.
\end{abstract}

Index terms: brown spot, Citrus reticulata, varietal resistance.

\footnotetext{
${ }^{1}$ (Trabalho 122-09). Recebido em: 19-05-2009. Aceito para publicação em: 27-04-2010.

${ }^{2}$ Centro APTA Citros Sylvio Moreira/IAC, CP 04, CEP 13490-970, Cordeirópolis-SP, e-mails: fernando@centrodecitricultura.br; mabastiane1@entrodecitricultura.br,katia@entrodecitricultura.br, rodrigostuart@entrodecitricultura.br

${ }^{3}$ Centro de Ciências Agrárias, Universidade Federal de São Carlos, CEP 13600-970, Araras-SP, e-mail: denisp@cca.ufscar.br

${ }^{4}$ Pós-Graduação - Agricultura Tropical e Subtropical, Instituto Agronômico, Rua Barão de Itapura, 1481, CP 28, CEP 13012-970, e-mail: franpcosta@yahoo.com.br
} 
O Brasil é o maior produtor mundial de citros e o maior exportador de suco concentrado de laranja. Apesar de ter a laranja-doce [Citrus sinensis (L.) Osbeck] como principal espécie produzida, várias espécies de tangerinas e alguns de seus híbridos merecem destaque nos pomares brasileiros, quando o destino é o mercado nacional de frutos in natura e apresenta potencial para exportação.

Nos pomares de tangerinas e híbridos, a mancha-marrom de alternária (MMA), causada pelo fungo Alternaria alternata, tem sido considerada a principal doença fúngica dessa espécie (PERES et al., 2003). A exemplo do que acontece no Brasil, a redução dos plantios de tangor Murcott (Citrus sinensis L. Osbeck x C. reticulata L. Blanco), variedade altamente suscetível (REIS et al., 2007) e a alta severidade da doença em regiões de clima úmido, onde o controle é muito difícil, tem ocasionado o abandono de plantios comerciais de variedades que se mostram altamente suscetíveis nos Estados Unidos, como a tangerina Dancy (C. reticulata) e alguns dos seus híbridos (TIMMER et al., 2000). Interessante que a tangerina Dancy é o parental da maioria dos híbridos, que são suscetíveis à mancha-marrom de alternária, possivelmente, embora não confirmado, também seja um dos parentais do tangor Murcott.

A mancha-marrom de alternária tem sido relatada na África do Sul (SWART et al., 1996), Espanha (VICENT et al., 2000), Itália (BELLA et al., 2001) e na Argentina (PERES et al., 2003), entre outros (BASTIANEL et al., 2005). No Brasil, a presença da mancha-marrom de Alternária foi confirmada nos Estados de Minas Gerais, Rio Grande do Sul, Rio de Janeiro e São Paulo, afetando seriamente os pomares formados pela tangerina Ponkan (Citrus reticulata Blanco) e tangor Murcott, principais variedades cultivadas no País, além da tangerina Dancy (GÓES et al., 2001; PERES et al., 2003; SPÓSITO et al., 2003). A presença da doença vem inviabilizando a produção, principalmente de tangor Murcott no Estado de São Paulo, fazendo com que muitos citricultores erradiquem seus pomares, devido ao elevado número de aplicações de fungicidas necessário e, consequentemente, ao drástico aumento no custo de produção, desestimulando o plantio de novas áreas.

O fungo, causador da doença, infecta, coloniza e reproduz-se em folhas, ramos e frutos, e sobrevive nos restos culturais presente na árvore ou caído no solo. A reprodução é caracterizada pela produção de esporos assexuais (conídios), que germinam sobre o tecido suscetível liberando ACT, uma toxina específica ao hospedeiro-tangerina, necrosando rapidamente os tecidos infectados. As condições ideais ocorrem com temperaturas em torno de 20 a $27^{\circ} \mathrm{C}, 10$ a 12 horas de molhamento contínuo, para a exibição dos sintomas, um ou dois dias após a germinação dos esporos (TIMMER et al., 2000).

Os sintomas ocorrem em ramos, frutos, tecidos verdes imaturos ou em fase de crescimento (KOHMOTO et al., 1993). Em folhas jovens, os sintomas iniciais caracterizam-se por pequenas manchas de cor marrom ou preta, circundadas ou não por halos amarelados que, posteriormente, se estendem, podendo abranger grande área da folha. As brotações novas, tanto vegetativas como reprodutivas, apresentam um aspecto de requeima no caule, culminando com a morte dos ponteiros e posterior envassouramento. Em ramos finos, ocorrem pequenas lesões corticosas com ou sem a presença de halo clorótico (AKIMITSU et al. 2003).

A maioria das variedades de tangerina Clementina e seus híbridos, bem como da tangerina Cleópatra, limas, limões, laranja-azeda e das laranjas-doces são resistentes à doença em condições de campo (GARDNER et al., 1986; KOHMOTO et al., 1991). Entretanto, em condições de inoculação artificial, os sintomas foram observados em laranja Valência, Shamouti e Washington Navel e limão Volkameriano para isolados provenientes de tangelo Minneola (SOLEL; KIMCHI, 1997).

A disseminação do fungo está altamente condicionada às condições ambientais. Portanto, em geral, são necessárias várias aplicações anuais de fungicidas para reduzir a severidade da doença, podendo chegar a 15 pulverizações (TIMMER et al., 2003), o que aumenta significativamente os custos de produção. Práticas culturais, como a formação de pomares em áreas com circulação de ar, maior espaçamento entre as plantas, cuidados com a adubação, principalmente a nitrogenada, que induz um grande crescimento vegetativo da planta, são recomendadas para diminuir a severidade da doença. Como o fungo só se multiplica em tecidos mortos, é recomendável a realização de podas de limpeza, geralmente realizadas no inverno, para eliminar restos culturais do pomar, para melhorar a aeração na planta (TIMMER et al., 2003).

A utilização de variedades resistentes nos pomares é uma das mais importantes estratégias de manejo das doenças de plantas. Dessa forma, a busca por novas variedades de tangerinas e seus híbridos, que apresentem maior resistêrncia à Alternaria alternata e, ao mesmo tempo, com valor de mercado, constituiu-se no objetivo deste trabalho.

Para tanto, cinquenta e quatro genótipos de tangerinas e seus híbridos foram avaliados quanto à suscetibilidade ou resistência à A. alternata, agente causal da mancha-marrom de Alternária, in vitro - em 
folhas destacadas, e in vivo - em plântulas obtidas de sementes, incluindo mexericas (Citrus deliciosa Ten.), tangores (C. reticulata Blanco x $C$. sinensis L. Osbeck), tangelos (C. reticulata $\times$ C. paradisi Macf.) e satsumas (C. unshui). Os experimentos foram conduzidos, respectivamente, em 2007 e 2008, no Centro APTA Citros Sylvio Moreira do Instituto Agronômico (IAC), no município de Cordeirópolis$\mathrm{SP}$, e os genótipos foram selecionados no Banco Ativo de Germoplasma de Citros (BAG) dessa mesma Instituição. Essa coleção é uma das maiores do mundo, contendo aproximadamente 350 diferentes genótipos do grupo das tangerinas.

Inicialmente, testes de patogenicidade foram realizados com nove isolados de A. alternata, provenientes de variedades de tangerinas, além de outro isolado de limão Rugoso (Citrus jambhiri Lush), utilizando-se de folhas jovens destacadas de tangor Murcott (aproximadamente $4 \mathrm{~cm}$ ), conforme o método descrito por Peever et al. (1999), com pequenas modificações.

De acordo com o método, utilizaram-se isolados monospóricos de A. alternata, obtidos de lesões típicas da doença em folhas ou frutos cultivados e preservados em meio de cultura BDA - (200 g batata, $20 \mathrm{~g}$ dextrose, $15 \mathrm{~g}$ Agar L $\mathrm{L}^{-1}$ ), contendo fungicida carbendazin (640 $\mathrm{mg} \mathrm{L}^{-1}$ do i.a.), para evitar a contaminação por outros fungos de esporos hialinos, a exemplo de Colletotrichum spp. Posteriormente, foram incubados a $27^{\circ} \mathrm{C}$, com fotoperíodo de 12 horas. $\mathrm{O}$ fungo $A$. alternata geralmente possui alto potencial para a degradação de carbendazin (SILVA et al., 1999), composto químico do grupo dos Benzimidazóis, facilitando seu isolamento na presença dessa substância.

Para induzir a produção de conídios - esporulação do fungo, colônias com 48 horas de idade foram transferidas para placas de Petri, contendo BDA, e incubadas nas mesmas condições do inóculo inicial, durante cinco dias. Em seguida, 50 discos (5 $\mathrm{mm}$ ) foram transferidos para placas contendo meio nutritivo para esporulação ( $30 \mathrm{~g}$ de $\mathrm{CaCO}_{3}, 20 \mathrm{~g}$ de

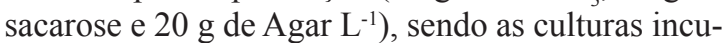
badas nas mesmas condições descritas anteriormente, por quatro dias.

Na sequência, com auxílio de uma espátula estéril, os conídios foram raspados da superfície da placa e ressuspendidos em água destilada esterelizada. A suspensão de conídios foi filtrada em duas camadas de gazes esterilizadas, para a remoção dos fragmentos miceliais, sendo a concentração ajustada para $10^{4}$ conídios $\mathrm{mL}^{-1}$, com auxílio de hemocitômetro.

Para a realização do teste de patogenicidade, folhas jovens de tangor Murcott (aproximadamente
$4 \mathrm{~cm}$ de comprimento), suscetível a A. alternata, foram coletadas no campo, lavadas em água corrente e acondicionadas em placas de Petri, contendo uma camada de papel-filtro e algodão umedecidos com água destilada e esterilizada. Em seguida, suspensões de inóculo ( $\left.2 \mathrm{~mL}_{\text {folha }}{ }^{-1}\right)$, de cada um dos isolados de A. alternata, foram pulverizadas (borrifador manual) sobre as folhas de tangor Murcott. Utilizaram-se quatro folhas por isolado, sendo essas incubadas em BOD (FANEM, 347CGD) a $27^{\circ} \mathrm{C}$, fotoperíodo de 12 horas, por três dias. Após isso, efetuou-se a avaliação, que consistiu na contagem do número de lesões por folha, sendo os valores de cada isolado submetidos à análise de variância, e as médias comparadas (Tukey a $5 \%$ de probabilidade), quando o teste $\mathrm{F}$ foi significativo.

Para a inoculação em folhas dos 54 genótipos em estudo (in vitro), utilizou-se a mesma metodologia descrita anteriormente. Após obtenção do inóculo, do isolado mais agressivo (A1-3), obtido no teste de patogenicidade, folhas jovens (um mês de idade) de cada genótipo estudado foram coletadas no campo, lavadas em água corrente, inoculadas com uma suspensão de $10^{4}$ esporos $\mathrm{mL}^{-1} \mathrm{e}$ incubadas em BOD a $27^{\circ} \mathrm{C}$, fotoperíodo 12 horas, durante dois dias.

As avaliações foram iniciadas 12 horas após inoculação, com observações da presença de lesões, sendo que o número por folha em cada genótipo foi aferido, dois dias após inoculação (48 horas), e as médias, submetidas ao teste estatístico de comparação Scott-Knot (5\%).

Para o ensaio in vivo - sementes extraídas de frutos de todos os genótipos foram semeadas em tubetes plásticos, contendo substrato específico para citros, sendo três plântulas de cada genótipo estudado transferidas para vasos (4L) e mantidas em casa de vegetação. Aos 90 dias, quando as plantas apresentavam tamanho superior a $30 \mathrm{~cm}$, efetuou-se uma poda, com intuito de forçar a brotação e, consequentemente, a formação de folhas jovens.

Após 35 dias, as plantas foram aspergidas com $2 \mathrm{~mL}$ de uma suspensão de $10^{4}$ conídios $\mathrm{mL}^{-1}$ (isolado A1-3), utilizando-se de borrifador manual, na face abaxial das folhas. Após a inoculação, as plantas foram mantidas em condições de câmara úmida e cobertas por sacos plásticos transparentes, durante 36 horas. As avaliações dos sintomas foram realizadas 72 horas após a inoculação e consistiram na contagem do número de lesões e na estimativa da área lesionada ( $\%$ da folha tomada pela doença), sendo essa realizada com auxílio de uma escala diagramática, com notas variando entre 0 e $6(0-0 \%$; $1-5 \% ; 2-15 \% ; 3-25 \%$ e, $4-35 \%$ ou mais de $35 \%$ de área lesionada). 
Os dados obtidos nos ensaios in vitro e in vivo foram submetidos à análise de variância, conforme delineamento proposto (inteiramente casualizado, com quatro repetições/folhas), e as médias, comparadas pelo teste de Scott-Knott, a 5\% de probabilidade, quando o teste $\mathrm{F}$ foi significativo.

Os testes de patogenicidade realizados com diversos isolados de A. alternata, inoculados em folhas destacadas de tangor Murcott, possibilitaram a seleção de um bastante agressivo (A1-3), quando comparado com os demais isolados que foram utilizados nas inoculações dos diversos genótipos, nos ensaios in vitro e in vivo. Esse isolado foi proveniente de frutos de tangelo Nova, um híbrido suscetível à mancha-marrom de alternária, e provocou grande número de lesões em folhas jovens de Murcott (26,6 lesões), diferindo significativamente da maioria dos isolados testados (Figura 1).

Como esperado, não foram observadas lesões nas folhas tratadas apenas com água (testemunha) nem nos inoculados com o isolado L2704, proveniente de limão Rugoso (Citrus jambhiri). Os demais isolados, provenientes do grupo das tangerinas, provocaram sintomas em folhas de Murcott. Esse resultado confirma o que está na literatura, mostrando o alto grau de especificidade entre isolados de tangerina (toxina ACT) e de limão Rugoso (toxina ACR) (KOHMOTO et al., 1979; GARDNER et al., 1985).

Grande variabilidade foi observada em resposta à inoculação de $A$. alternata dentro do grupo das tangerinas e seus híbridos, tanto in vitro quanto in vivo (Tabela 1). Dos genótipos avaliados in vitro, apenas 22 não apresentaram sintomas da doença, onde as condições são ideais. Por outro lado, nas inoculações in vivo, um maior número de genótipos mostrou-se resistente (32). Folhas avaliadas aos dois e três dias após inoculações in vitro e in vivo, respectivamente, foram mantidas até o quinto dia.

$\mathrm{O}$ fato de ter sido observado, no ensaio in vivo, menor número de genótipos com folhas sintomáticas, evidenciou maior resistência das plantas nessas condições. Segundo Solel e Kimchi (1998), maior resistência dos tecidos das plantas suscetíveis está associada ao avanço da maturidade dos mesmos. De acordo com Whiteside (1976), os frutos são suscetíveis até $4 \mathrm{~cm}$ de diâmetro, podendo estender-se até 5-6 cm, dependendo da espécie (VICENT et al., 2004; REIS et al., 2006).

Os resultados obtidos nesses estudos (Tabela 1) sugerem que o grupo das satsumas [C. unshiu (Mark.) Marc.] é muito resistente à mancha-marrom de alternária, uma vez que não foram observados sintomas, nos ensaios in vitro e in vivo, quando inoculadas com um isolado altamente agressivo do fungo. Reis et al. (2007) avaliaram dez variedades desse grupo, os quais também se mostraram resistentes à $A$. alternata em inoculações similares (in vitro e in vivo). Por outro lado, quando os testes foram realizados em folhas muito jovens (2-4 cm), todos os genótipos apresentaram sintomas da doença. Kohmoto et al. (1991) também não observaram sintomas da doença em inoculações em satsumas, evidenciando a resistência desse grupo de tangerinas.

Genótipos assintomáticos também foram observados no grupo das mexericas, onde apenas dois genótipos: a Pernambucana 2 e a Córsega foram suscetíveis dentre as estudadas. No grupo das tangerinas e híbridos, destacaram-se Ellendale, Szinkon, Cravo, Fremont e Thomas. Reis et al. (2007) descreveram resistência à mancha-marrom de alternária (folhas acima de $4 \mathrm{~cm}$ de comprimento) nos genótipos Fremont, Encore e Fallglo, em inoculações in vivo e in vitro. Vicent et al. (2004) observaram resistência dos híbridos Ellendalle e Ortanique oriundos de cruzamento entre $C$. sinensis x $C$. reticulata, quando submetidos à inoculação de três isolados de A. alternata. Esses resultados confirmam a existência de resistência ao fungo dentro desse grupo de tangerinas.

A confirmação da resistência à A. alternata em Fremont e Thomas aumenta a importância econômica desses genótipos, principalmente porque já vêm sendo estudados por apresentarem boas características agronômicas. A variedade Thomas lembra muito o tangor Murcott, por apresentar casca aderente, muitas sementes e maturação tardia (outubro-novembro), além de ser compatível com o porta-enxerto citrumelo Swingle (PIO; MINAMI, 2002; BARBASSO et al., 2005). Trabalhos recentes também descrevem bons aspectos da variedade Fremont, que possui coloração da casca alaranjado-forte, maturação em meia-estação (maio-julho), porém os frutos permanecem fixos no pé por mais tempo, quando comparado à Ponkan (PIO et al., 2006, NÚÑ̃ES et al., 2007). A resistência à mancha-marrom de alternária em frutos, observada em campo, dos genótipos Thomas e Fremont, é relatada por Azevedo et al. (2007) e Feichtenberger et al. (2005), respectivamente.

Dentre os genótipos sintomáticos obtidos nesta pesquisa, destacaram-se alguns já descritos na literatura como altamente suscetíveis em outros países, tais como a Dancy e híbridos diretos ou indiretos (tangelos Minneola, Seminole, Orlando e Osceola), a tangerina Ponkan e o tangor Murcott (PEEVER et al., 2000). Especificamente, no grupo das tangerinas Ponkans, os genótipos Sul da África, Empress, Green Rind, Span Americana, Ponkan 1, 2, 4 e 7, casca-verde 
1 e 2 e Muscia não foram encontrados materiais resistentes, porém o genótipo Ponkan casca-verde 2 não apresentou sintomas, quando inoculado sob condições in vivo.

De fato, alguns autores, baseando-se no padrão de suscetibilidade dos genótipos de citros e seus híbridos, à doença, sugeriram que ela foi herdada do parental tangerina Dancy, como uma característica dominante, sendo, portanto, a resistência da mancha-marrom das tangerinas um caráter recessivo (KOHMOTO et al., 1991). Entretanto, a resposta diferenciada de híbridos bastante próximos sugere que outros genes podem estar envolvidos nessa interação (PEEVER et al., 2000) e para confirmar essa especulação oriunda de observações no campo, é preciso estabelecer experimentos genéticos específicos.

A maioria dos genótipos apresentou maior suscetibilidade, caracterizada pelo maior número de lesões nas inoculações in vitro, do que nas inoculaçôes in vivo (Tabela 1). Esse resultado era esperado, uma vez que, em com condições ideais e controladas, o fungo tem melhores condições de proliferar-se. Esse fato, também foi observado por Reis et al. (2007), avaliando diversas variedades do grupo das tangerinas, que exibiram número de lesões em folhas inoculadas in vivo, sempre inferior do que quando em folhas destacadas em laboratório. Menor expressão dos sintomas nas folhas in vivo, em campo ou estufa, pode estar relacionada à alternância de luminosidade, temperatura e condições de umidade, que são plenamente controladas em ensaios in vitro.

A variação encontrada no grau de resistência e suscetibilidade a Alternaria alternata é comum em testes de patogenicidade envolvendo espécies cítricas, para esse fungo. Vicent et al. (2004) também encontraram variação na suscetibilidade entre os genótipos testados e relataram que a variação pode ser influenciada pelas condições experimentais. Por isso, neste trabalho, foram utilizados tipos de ensaios, in vitro e in vivo, seguindo método consagrado para testar a resistência de espécies cítricas a $A$. alternata descrito por Peever et al. (2000).

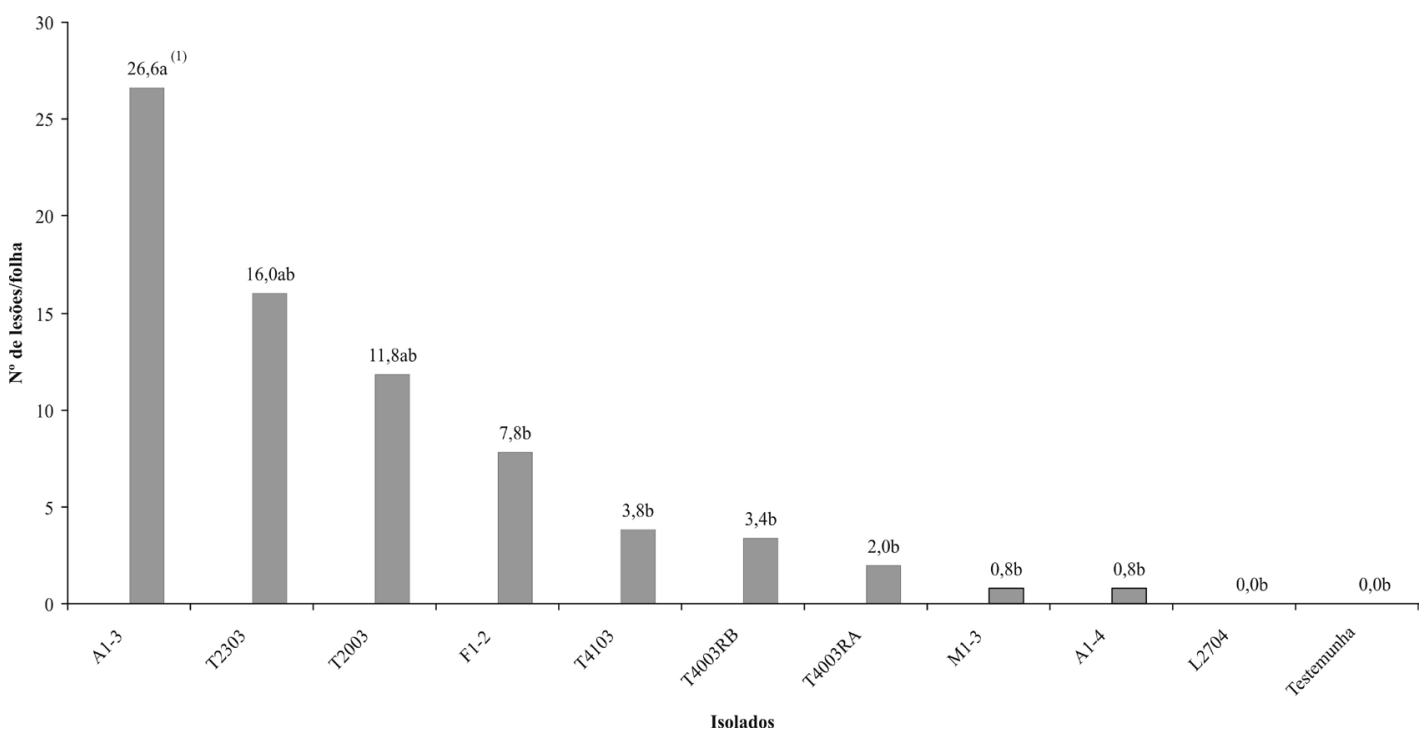

FIGURA 1 - Número médio de lesões de mancha-marrom de Alternária/folha jovem de tangor Murcott, inoculadas in vitro, com os diferentes isolados de A. alternata. 
TABELA 1 - Reação dos genótipos de tangerinas e híbridos quanto à suscetibilidade e resistência à Alternária alternata, quando expostas a inoculação in vitro e in vivo $(2007,2008)$.

\begin{tabular}{|c|c|c|c|}
\hline Genótipos & Inoculação in vitro & Inoculas & ão in vivo \\
\hline 1. Tangerinas (C. reticulata Blanco) & $N^{0}$ de lesões/folha & $N^{0}$ de lesões/folha & (\% área lesionada) \\
\hline Sul da África & $320,0 \mathrm{a}^{*}$ & $26,0 \mathrm{c}$ & $32,0 \mathrm{a}$ \\
\hline Empress & $226,7 \mathrm{~b}$ & $14,0 \mathrm{~d}$ & $8,0 \mathrm{c}$ \\
\hline Green Rind & $166,7 \mathrm{~b}$ & $43,0 \mathrm{~b}$ & $15,0 \mathrm{~b}$ \\
\hline Creola & $83,3 \mathrm{c}$ & $24,0 \mathrm{c}$ & $22,0 \mathrm{a}$ \\
\hline Span Americana & $65,0 \mathrm{c}$ & $2,0 \mathrm{e}$ & $3,0 \mathrm{~d}$ \\
\hline Dancy & $64,0 \mathrm{c}$ & $64,0 \mathrm{a}$ & $31,7 \mathrm{a}$ \\
\hline Ponkan 2 & $60,0 \mathrm{c}$ & $3,0 \mathrm{~d}$ & $8,0 \mathrm{c}$ \\
\hline Weshart & $53,0 \mathrm{c}$ & $31,0 \mathrm{c}$ & $28,0 \mathrm{a}$ \\
\hline Ponkan 7 & $46,7 \mathrm{c}$ & $3,0 \mathrm{~d}$ & $11,7 \mathrm{~b}$ \\
\hline Ponkan 1 & $46,7 \mathrm{c}$ & $18,0 \mathrm{c}$ & $8,0 \mathrm{c}$ \\
\hline Ponkan casca-verde 1 & $36,7 \mathrm{c}$ & $9,0 \mathrm{~d}$ & $5,0 \mathrm{c}$ \\
\hline Muscia & $24,7 \mathrm{~d}$ & $19,8 \mathrm{c}$ & $8,0 \mathrm{c}$ \\
\hline Szwinkon & $23,3 \mathrm{~d}$ & $25,0 \mathrm{c}$ & $28,0 \mathrm{a}$ \\
\hline Ponkan 4 & $2,0 \mathrm{e}$ & $5,0 \mathrm{~d}$ & $11,7 \mathrm{~b}$ \\
\hline Emperor & $4,0 \mathrm{e}$ & $1,0 \mathrm{e}$ & $5,0 \mathrm{c}$ \\
\hline Cravo & $3,7 \mathrm{e}$ & $0,0 \mathrm{e}$ & $0,0 \mathrm{~d}$ \\
\hline Vermelha 12 & $3,7 \mathrm{e}$ & $0,0 \mathrm{e}$ & $0,0 \mathrm{~d}$ \\
\hline Vermelha & $3,0 \mathrm{e}$ & $0,0 \mathrm{e}$ & $0,0 \mathrm{~d}$ \\
\hline Ponkan casca-verde 2 & $1,3 \mathrm{e}$ & $0,0 \mathrm{e}$ & $0,0 \mathrm{~d}$ \\
\hline Ellendale early & $0,3 \mathrm{e}$ & $0,0 \mathrm{e}$ & $0,0 \mathrm{~d}$ \\
\hline Ellendale late & $0,0 \mathrm{e}$ & $0,0 \mathrm{e}$ & $0,0 \mathrm{~d}$ \\
\hline Ellendale & $0,0 \mathrm{e}$ & $0,0 \mathrm{e}$ & $0,0 \mathrm{~d}$ \\
\hline King x Dancy & $0,0 \mathrm{e}$ & $0,0 \mathrm{e}$ & $0,0 \mathrm{~d}$ \\
\hline Hansen & $0,0 \mathrm{e}$ & $0,0 \mathrm{e}$ & $0,0 \mathrm{~d}$ \\
\hline Wilking & $0,0 \mathrm{e}$ & $0,0 \mathrm{e}$ & $0,0 \mathrm{~d}$ \\
\hline Vermelha 17 & $0,0 \mathrm{e}$ & $0,0 \mathrm{e}$ & $0,0 \mathrm{~d}$ \\
\hline Ladu x Szinkon & $0,0 \mathrm{e}$ & $0,0 \mathrm{e}$ & $0,0 \mathrm{~d}$ \\
\hline Szinkon & $0,0 \mathrm{e}$ & $0,0 \mathrm{e}$ & $0,0 \mathrm{~d}$ \\
\hline Szinkon x Tizon & $0,0 \mathrm{e}$ & $0,0 \mathrm{e}$ & $0,0 \mathrm{~d}$ \\
\hline Szinkon x Batangas & $0,0 \mathrm{e}$ & $0,0 \mathrm{e}$ & $0,0 \mathrm{~d}$ \\
\hline Fremont & $0,0 \mathrm{e}$ & $0,0 \mathrm{e}$ & $0,0 \mathrm{~d}$ \\
\hline Thomas & $0,0 \mathrm{e}$ & $0,0 \mathrm{e}$ & $0,0 \mathrm{~d}$ \\
\hline 2. Tangelos (C. reticulata Blanco x C & paradisi Macfad.) & & \\
\hline Orlando & $300,0 \mathrm{a}$ & $1,0 \mathrm{e}$ & $3,0 \mathrm{~d}$ \\
\hline Osceola & $61,7 \mathrm{c}$ & $29,0 \mathrm{c}$ & $28,0 \mathrm{a}$ \\
\hline Seminole & $48,3 \mathrm{c}$ & $27,0 \mathrm{c}$ & $18,0 \mathrm{a}$ \\
\hline Nova & $46,7 \mathrm{c}$ & $8,0 \mathrm{~d}$ & $10,0 \mathrm{c}$ \\
\hline Mineola & $38,3 \mathrm{~d}$ & $34,0 \mathrm{~b}$ & $28,0 \mathrm{a}$ \\
\hline 3. Tangores $[C$. reticulta Blanco $\times$ C. & sinensis (L.) Osbeck] & & \\
\hline Dweet & $50,0 \mathrm{c}$ & $49,0 \mathrm{~b}$ & $8,0 \mathrm{c}$ \\
\hline Murcott & $30,3 \mathrm{~d}$ & $32,0 \mathrm{c}$ & $8,0 \mathrm{c}$ \\
\hline Temple & $4,3 \mathrm{e}$ & $0,0 \mathrm{e}$ & $0,0 \mathrm{~d}$ \\
\hline Temple 3 & $2,3 \mathrm{e}$ & $0,0 \mathrm{e}$ & $0,0 \mathrm{~d}$ \\
\hline Temple 2 & $1,7 \mathrm{e}$ & $0,0 \mathrm{e}$ & $0,0 \mathrm{~d}$ \\
\hline Temple 1 & $0,0 \mathrm{e}$ & $0,0 \mathrm{e}$ & $0,0 \mathrm{~d}$ \\
\hline 4. Mexericas (C. deliciosa Ten.) & & & \\
\hline Pernambucana 2 & $18,7 \mathrm{~d}$ & $0,0 \mathrm{e}$ & $0,0 \mathrm{~d}$ \\
\hline Córsega & $0,7 \mathrm{e}$ & $0,0 \mathrm{e}$ & $0,0 \mathrm{~d}$ \\
\hline Siracusa & $0,0 \mathrm{e}$ & $0,0 \mathrm{e}$ & $0,0 \mathrm{~d}$ \\
\hline Paiva & $0,0 \mathrm{e}$ & $0,0 \mathrm{e}$ & $0,0 \mathrm{~d}$ \\
\hline 114412 & $0,0 \mathrm{e}$ & $0,0 \mathrm{e}$ & $0,0 \mathrm{~d}$ \\
\hline 1060 & $0,0 \mathrm{e}$ & $0,0 \mathrm{e}$ & $0,0 \mathrm{~d}$ \\
\hline Tardia da Sicília & $0,0 \mathrm{e}$ & $0,0 \mathrm{e}$ & $0,0 \mathrm{~d}$ \\
\hline 5. Satsumas $[C$. unshiu (Mark.) Mar & & & \\
\hline Japão & $0,0 \mathrm{e}$ & $0,0 \mathrm{e}$ & $0,0 \mathrm{~d}$ \\
\hline Wase & $0,0 \mathrm{e}$ & $0,0 \mathrm{e}$ & $0,0 \mathrm{~d}$ \\
\hline Owari & $0,0 \mathrm{e}$ & $0,0 \mathrm{e}$ & $0,0 \mathrm{~d}$ \\
\hline Sem sementes & $0,0 \mathrm{e}$ & $0,0 \mathrm{e}$ & $0,0 \mathrm{~d}$ \\
\hline
\end{tabular}

Sem sementes

*médias seguidas de mesma letra, na coluna, não diferem entre si (Scott-Knott, 5\%). 


\section{CONCLUSÃO}

1-Há grande diferença de suscetibilidade a Alternaria alternata, para lesões foliares, entre genótipos do grupo das tangerinas.

2-Os grupos satsumas, mexericas e algumas tangerinas são resistentes a $A$. alternata.

\section{AGRADECIMENTOS}

À Fundação de Amparo à Pesquisa do Estado de São Paulo (FAPESP), pelo apoio financeiro para a realização do trabalho (Processo: 06/55585-0), e ao Programa Institucional de Bolsas de Iniciação Científica do Conselho Nacional de Desenvolvimento Científico do Instituto Agronômico (PIBIC/CNPq/ IAC), pela concessão de bolsa ao segundo autor.

\section{REFERÊNCIAS}

AKIMITSU, K.; PEEVER, T.L.; TIMMER, L.W. Molecular, ecological and evolutionary approaches to undertanding Alternaria diseases of citrus. Molecular Plant Pathology, Sheffield, v.4, p.435-436, 2003.

AZEVEDO, F.A.; PIO, R.M.; DE NEGRI, J.D.; WIGMAN, M.; POLYDORO, D.A.; FEICHTENBERGER, E.; SPOSITO, M.B. Thomas: variedade de tangerina resistente à mancha-marrom de alternaria. In: CONGRESSO PAULISTA DE FITOPATOLOGIA, 30., 2007, Jaboticabal. Anais... Jaboticabal: UNESP, 2007. p.280-281.

BARBASSO, D.V.; PIO, R.M.; CARVALHO, S.A. Compatibilidade de variedades e híbridos de tangerinas enxertadas em citrumelo 'Swingle'. Laranja, Cordeirópolis, v.26, p.59-67, 2005.

BASTIANEL, M.; AZEVEDO, F.A.; CRISTOFANI, M.; MACHADO, M.A. Mancha-marrom: nova doença nos pomares de tangerineiras. Laranja, Cordeirópolis, v.27, p.323-336, 2005.

BELLA, P.; GUARINO, C.; LA ROSA, R.; CATARA, A. Severe infections of Alternaria spp on a mandarin hybrid. Journal of Plant Pathology, Bari, v.83. p.231, 2001.

FEICHETENBERGER, E.; SPÓSITO, M.B.; PIO, R.M.; CASTRO, J.L. Seleção de híbridos de tangerinas e híbridos de citros para tolerância à manchamarrom de Alternária (Alternaria alternata Keissler). Citricultura Atual, Cordeirópolis, v.8, p.8-10, 2005
GARDNER, J.M.; KONO, Y.; CHANDLER, J.L. Biossay and host-selectivity of Alternaria citri toxins affecting rouge lemon and mandarins. Physiology Molecular Plant Pathology, East Lansing, v.29, p.293-304, 1986.

GARDNER, J.M.; KONO, Y.; TATUM, J.H.; SUZUKI, Y.; TAKEUCHI, S. Structure of major component of ACRL toxins, host-specific phytotoxic compound produced by Alternaria citri. Agricultural and Biological Chemistry, Brunkyoku, v.49, p.1235-1238, 1985.

GOES, A.; MONTES DE OCA, A.G.; REIS, R.F. Ocurrencia de la mancha de alternaria em mandarina 'Dancy' en el Estado de Rio de Janeiro. Fitopatologia Brasileira, Brasília, v.26, p.386, 2001.

KOHMOTO, K.; AKIMITSU, K.; OTANI, H. Correlation of resistance and suscetibility of citrus to Alternaria alternata with sensitivity to host-specific toxins. Phytopathology, St Paul, v.81, p.719-722, 1991.

KOHMOTO, K.; ITOH, Y.; SHIMOMURA, N.; KONDOH, Y.; OTANI, H.; KODAMA, M.; NISHIMURA, S.;NAKATSUKA, S. Isolation and biological activities of two host-specific toxins from the tangerine pathotype of Alternaria alternata. Phytopathology, St Paul, v.83, p.495-502, 1993.

KOHMOTO, K.; SCHEFFER, R.P.; WHITESIDE, J.O. Host-selective toxins from Alternaria citri. Phytopathology, St Paul, v.69, p.667-671, 1979.

NÚÑEZ, E.E.; MOURÃO FILHO, F.A.A.; STUCHI, E.S. Desenvolvimento vegetativo, produção e qualidade de frutos da tangerina 'Fremont' sobre quatro porta-enxertos. Revista Brasileira de Fruticultura, Jaboticabal, v.29, p.308-312, 2007.

PEEVER, T.L.; CANILHOS, Y.; OLSEN, L.; IBÁÑEZ, A.; LIU, Y.C.; TIMMER, L.W. Population genetic structure and host specificity of Alternaria spp causing brown spot of Minneola tangelo and rough lemon in Florida. Phytopathology, St Paul, v.89, p.851-860, 1999.

PEEVER, T.L.; OLSEN, L.; IBÁÑEZ, A.; TIMMER, L.W. Genetic diferentiation and host specificity among populations of Alternaria spp causing brown spot of grapefruit and tangerine $\mathrm{x}$ grapefruit hybrids in Florida. Phytopathology, St Paul, v.90, p.407414, 2000. 
PERES, N.A.R.; AGOSTINI, J.P.; TIMMER, L.W. Outbreaks of Aternaria brown spot of citrus in Brazil and Argentina. Plant Disease, St Paul ,v.87, p.750, 2003.

PIO, R.M.; AZEVEDO, F.A.; DE NEGRI, J.D.; FIGUEIREDO, J.O.; GARCIA, V.X.P. Características da variedade Fremont quando comparadas com as das tangerinas Ponkan e Clementina Nules. Revista Brasileira de Fruticultura,Jaboticabal, v.28, p.222226, 2006.

PIO, R.M.; MINAMI, K. Caracterização dos frutos das variedades de tangerinas Sul da África e Thomas. Laranja, Cordeirópolis, v. 23, n.473-488, 2002.

REIS, R.F.; ALMEIDA, T.F.; STUCHI, E.S.; GOES, A. Susceptibility of citrus species to Alternaria alternata, the causal agent of the Alternaria brown spot. Scientia Horticulturae, Wageningen, v.113, p.336-342., 2007.

REIS, R.F.; GOES, A.D.E.; MONDAL, S.M.; TIMMER, L.W. Effectiveness of fungicides and susceptibility of fruit and leaves of tangerine, tangor and tangelos to infection by Alternaria alternata, the cause of brown spot. Summa Phytopathologica, Botucatu, v.32, p.1-12, 2006.

SILVA, C.M.M.S; MELO, I.S.; MAIA, A.H.N.; ABAKERLI, R.B. Isolamento de fungos degradadores de carbendazim. Pesquisa Agropecuária Brasileira, Brasília, v.34, p.1255-1264, 1999.

SOLEL, Z.; KIMCHI, M. Histopathology of infection of Minneola tangelo by Alternaria alternata pv citri and the effect of host and environmental factors on lesion development. Journal of Phytopathology, Oxford, v.146, p.557-561, 1998.

SOLEL, Z.; KIMCHI, M. Susceptibility and resistance of citrus genotypes to Alternaria alternata pv citri. Journal of Phytopathology, Oxford, v.145, p.389-391, 1997.
SPÓSITO, M.B.; FEICHTENBERGER, E.; PIO, R.M.; CASTRO, J.L.; RENAUD, M.S.A. Ocorrência de mancha-marrom de Alternária em diferentes genótipos de citros nos Estados de Minas Gerais, São Paulo e Rio Grande do Sul. Fitopatologia Brasileira, Botucatu, v.28, p.231, 2003.

SWART, S.H.; WINGFIELD, M.J.; SWART, W.J.; SCHUTTE, G.C. Brown spot of Minneola tangelo and efficacy of fungicidal sprays programmes for disease control in South Africa. Proceedings of the International Society of Citriculture, South Africa, v.1, p.379-384, 1996.

TIMMER, L.W.; PEEVER, T.L.; SOLEL, Z.; AZUYA, K. Alternaria diseases of citrus-novel pathosystems. Phytopathologia Mediterranea, Bologna, v.42, p.99-112, 2003.

TIMMER, L.W.; SOLEL, Z.; OROZCO-SANTOS, M. Alternaria Brown Spot of mandarines In: TIMMER, L.H.; GARNSEY, S.M.; GRAHAM, J.H. (Ed.). Compendium of citrus diseases 2. ed. Minnesota: APS Press ST Paul, 2000. p.19.

VICENT, A.; ARMENGOL, J.; SALLES, R.; ALFARO-LASSALA, F.; GARCIA- JIMÉNEZ, J. First report of Alternaria Brown Spot of citrus in Spain. Plant Disease, St Paul, v.84, p.1044, 2000.

VICENT, A.; BADAL, J.; ASENSI, M.J.; SANZ, N.; ARMENGOL, J.; GARCIA-JIMENEZ, J. Laboratory evaluation of citrus cultivars susceptibility and influence of fruit size on Fortune mandarin to infection by Alternaria alternata pv citri. European Journal of Plant Pathology, Dordrecht, v.110, p.245-251, 2004.

WHITESIDE, J.O. A newly recorded Alternaria induced Brown Spot disease on Dancy tangerines in Florida. Plant Disease Reporter, St Paul, v.60, p.326-329, 1976. 